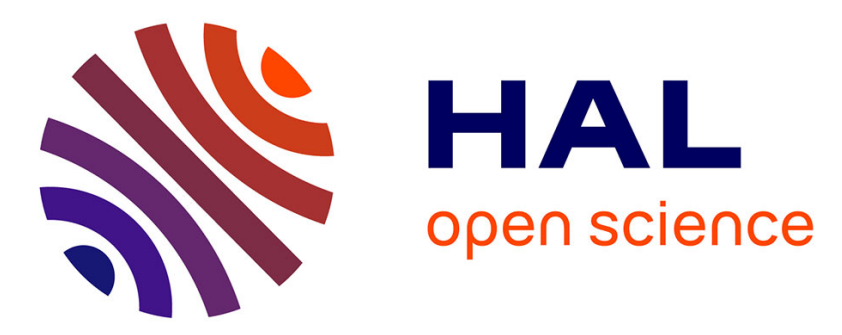

\title{
On Bisection Continuous Collision Checking Method: Spherical Joints and Minimum Distance to Obstacles
}

Sonny Tarbouriech, Wael Suleiman

\section{To cite this version:}

Sonny Tarbouriech, Wael Suleiman. On Bisection Continuous Collision Checking Method: Spherical Joints and Minimum Distance to Obstacles. ICRA 2018 - IEEE International Conference on Robotics and Automation, May 2018, Brisbane, Australia. pp.7613-7619, 10.1109/ICRA.2018.8463199 . hal01816846

\section{HAL Id: hal-01816846 \\ https://hal.science/hal-01816846}

Submitted on 15 Jun 2018

HAL is a multi-disciplinary open access archive for the deposit and dissemination of scientific research documents, whether they are published or not. The documents may come from teaching and research institutions in France or abroad, or from public or private research centers.
L'archive ouverte pluridisciplinaire HAL, est destinée au dépôt et à la diffusion de documents scientifiques de niveau recherche, publiés ou non, émanant des établissements d'enseignement et de recherche français ou étrangers, des laboratoires publics ou privés. 


\title{
On Bisection Continuous Collision Checking Method: Spherical Joints and Minimum Distance to Obstacles
}

\author{
Sonny Tarbouriech and Wael Suleiman
}

\begin{abstract}
In this paper, we adapt the Continuous Collision Detection (CCD) method proposed in [1] to efficiently handle the case of spherical and two revolute joints, this kind of joints is very common in modern robotic systems. The new formulations provide more tight motion bounds, thus increase the success rate of checking collision-free paths. We also propose an extension to get the minimum distance to obstacles along a path, this information is primordial as it allows samplingbased motion planning techniques to sort collision-free paths according to their minimum clearance.

We have integrated our implementation into a samplingbased motion planning technique and validated it through simulation and on the real Baxter research robot. The experiments revealed that the method not only does not miss any collision between the robot and the obstacles, but also the minimum distance extension provides the path with the maximum clearance at no additional computational cost.
\end{abstract}

\section{INTRODUCTION}

Collision checking is an essential step in motion planning as it ensures the path to be collision-free, and it has been a very active research topic during the last two decades. However, this is also one of the most computationally expensive operations in the process of finding a clear path, especially in a complex environment.

The main challenge relies on determining whether the continuous path between two states in the Configurationspace (C-space) is in collision or not. In sampling-based algorithms, a commonly used method consists in testing individual configurations by linear interpolation between the states. The principal cumbersome of such an approach is that a compromise must be made when selecting the number of samples. Decreasing it leads to a loss of accuracy and could yield to a collision omission. On the other hand, increasing it could as well increase the computational time for collision checking, which is practically inefficient strategy in situations where the robot is far from obstacles.

In the context of unstructured environments where the safety of the robot, and possibly of humans interacting with it, is a priority, a computationally efficient method for exact collision checking is primordial.

The guarantee of a collision-free path has been introduced by Quinlan in [2] and is obtained by using overlapping free bubbles in the C-space along a segment. This concept was then integrated in several motion planning strategies [3], [4].

S. Tarbouriech and W. Suleiman are with Electrical and Computer Engineering Department, Faculty of Engineering, Université de Sherbrooke, 2500, boul. Universite, Sherbrooke, Quebec (Canada). \{Sonny.Tarbouriech@USherbrooke.ca, Wael.Suleiman@USherbrooke.ca\}

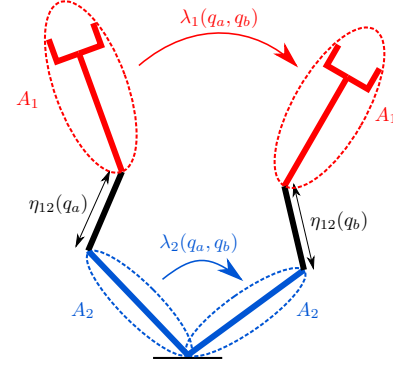

(a) Self-collision (b) Collision with an external object

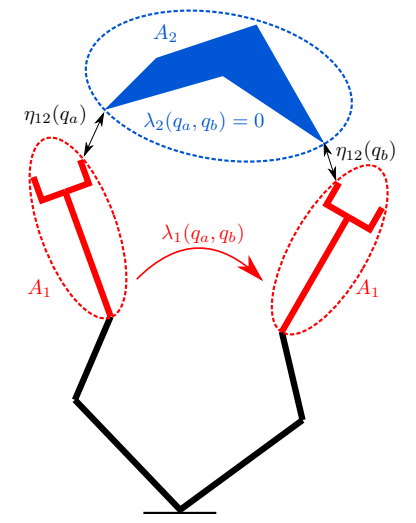

Fig. 1. Example: Two types of collision analysis for a 3-DOF robotic arm

The concept of Continuous Collision detection (CCD) has also received a lot of attention as it guarantees a path to be collision-free. A CCD algorithm for articulated models has been proposed in [5], it is able to compute the time of contact and contact locations. A fast CCD algorithm for articulated models using Taylor models and temporal culling has been proposed in [6], and another fast and exact CCD algorithm which uses properties of the Bernstein basis and Bézier curves has been presented in [7]. Moreover, an algorithm that performs CCD for polygon-soup models undergoing rigid motion at highly interactive rates is presented in [8]. CCD algorithms have been also used in local planning in samplingbased motion planning [1], [9], [10].

In this paper, we are interested in the category of CCD for motion planning, mainly the CCD method proposed in [1] and [10], this method is overviewed in Section II.

Our contributions are twofold:

- Adapting the CCD method in [1] to the case of two revolute and spherical joints; hence proving more tight motion bounds. This contribution is presented in Sections III-A and III-B.

- Proposing an extension to [1] to get information about the minimum distance to obstacles along a collision-free path. The detail of this contribution is given in Section IV.

We have also validated our algorithm on a Baxter research robot through simulation and real experiments. The experimental results are analyzed in Section V. 


\section{Adaptive Bisection Continuous Collision CHeCKING METHOD}

The main idea behind this method is to establish a sufficient condition of collision-free by computing the geometric path of rigid bodies in the workspace. Lemma 1 in [1] states that a sufficient condition to guarantee that two rigid objects, $A_{1}$ and $A_{2}$, do not collide at any configuration $q$ located on the path segment $\pi$, which is joining two configurations $q_{a}$ and $q_{b}$, is to verify the following inequality:

$$
\lambda_{1}\left(q_{a}, q_{b}\right)+\lambda_{2}\left(q_{a}, q_{b}\right)<\eta_{12}\left(q_{a}\right)+\eta_{12}\left(q_{b}\right)
$$

where $\eta_{12}\left(q_{i}\right)$ is the minimum distance between objects $A_{1}$ and $A_{2}$ for a given configuration $q_{i}$, and $\lambda_{i}\left(q_{a}, q_{b}\right)$ refers to the maximum Euclidean displacement of all the points in an object $A_{i}$ along the path segment $\pi$.

In the case of a robotic arm, we will focus on the two following situations (represented in Fig. 1):

- Self-collision detection: Both objects $A_{1}$ and $A_{2}$ are distinct links of the robot.

- Detection of collisions with the environment: $A_{1}$ is a link of the robot and $A_{2}$ is an obstacle in the world. As it is an off-line analysis, we assume that objects in the environment are static. Consequently, in this case, $\lambda_{2}\left(q_{a}, q_{b}\right)=0$ in (1).

To make a complete collision check for a given path segment, we have to evaluate all pairs of elements which could potentially collide using (1). If the test succeeds for a pair, we ensure that those two objects do not collide and we move on to the next pair. If the inequality is not verified, we cannot affirm that the path is collision-free. However, that does not mean a collision necessarily exists, but more tests are needed. To this end, we add an intermediate state on the path segment $\pi$. This new configuration divides the initial segment in two parts. If a collision is detected for this state, the motion is then invalid. Otherwise, we evaluate in the same manner each subsegment independently for the same two objects. If both subsegments satisfy the inequality, the path segment is collision-free for the given pair of elements, if not, we repeat the process for the subsegments that failed the test. More details about the method are given in [1]. The procedure of applying this method to a multi-DOF robotic arm is also presented in [1].

\section{DisplaCEMENTS IN C-SPACE AND WORKSPACE}

To apply the method in Section II, the term $\lambda_{i}\left(q_{a}, q_{b}\right)$ in (1) should be computed to verify the inequality constraint. Computing the exact displacement of a multi-DOF robotic arm between two configurations could be a complex process, and therefore a time-consuming operation. However, an upper bound of this distance can be quickly figured out by only considering the displacement range of each joint and the dependency between the links movements.

The original method in [1] considers the case of a single link $\mathbb{L}$ of length $L$, which is controlled by a revolute joint.

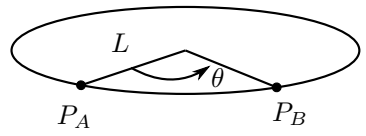

Fig. 2. Displacement of a link controlled by one joint

In this case, an elementary joint variation $d \theta$ leads to a traveling distance for the link of

$$
d \lambda=L d \theta
$$

Fig. 2 illustrates the total displacement of the link between configurations $q_{a}$ and $q_{b}$. The point $P \in \mathbb{L}$ which travels the longest distance is located at the end of the link and moves from $P_{A}$ to $P_{B}$. The total length $s$ is:

$$
\lambda=\int_{\theta^{a}}^{\theta^{b}} L d \theta=L\left(\theta^{b}-\theta^{a}\right)
$$

\section{A. Two Revolute Joints Case}

The case where two revolute joints control the same link, as illustrated in Fig. 3, is particularly interesting for modern redundant robotic arms, and it is the case for the Baxter research robot.

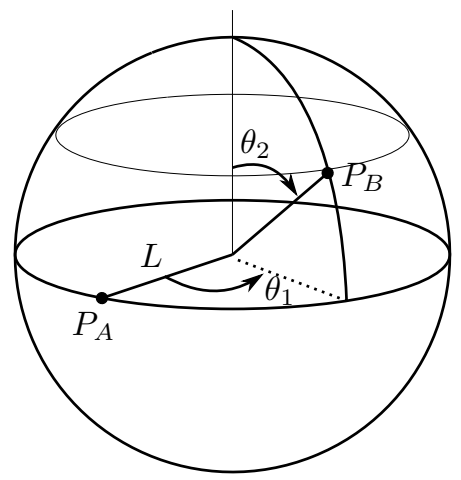

Fig. 3. Displacement of a link controlled by two revolute joints

By using the original formula proposed in [1], the upper limit of the displacement would be:

$$
\bar{\lambda}^{*}=L\left(\left|\theta_{1}^{b}-\theta_{1}^{a}\right|+\left|\theta_{2}^{b}-\theta_{2}^{a}\right|\right)
$$

It is clear that this over-estimation could increase the probability that a segment fails the collision-free test in (1), and as a result a sub-segmentation is required.

To compute a more tighter upper-bound, let us start by computing an elementary displacement of a point $P$ situated at the end of the link:

$$
d \lambda=L \sqrt{\left(d \theta_{2}\right)^{2}+\sin ^{2} \theta_{2}\left(d \theta_{1}\right)^{2}}
$$

The total traveled distance can be defined by:

$$
\lambda=\int_{\theta_{1}^{a}}^{\theta_{1}^{b}} \int_{\theta_{2}^{a}}^{\theta_{2}^{b}} L \sqrt{\left(d \theta_{2}\right)^{2}+\sin ^{2} \theta_{2}\left(d \theta_{1}\right)^{2}}
$$


By linearly interpolating between the configurations $q_{a}$ and $q_{b}$, we obtain $d \theta_{2}=\beta d \theta_{1}$, where $\beta$ is a constant. Let us suppose that $\sin ^{2} \theta_{2}^{*}=\max _{\theta_{2}^{a} \leq \theta_{2} \leq \theta_{2}^{b}}\left(\sin ^{2} \theta_{2}\right)$, thus the integral (6) can be upper bounded by a simple integral:

$$
\begin{aligned}
\lambda \leq \bar{\lambda} & =\int_{\theta_{1}^{a}}^{\theta_{1}^{b}} L \sqrt{\beta^{2}+\sin ^{2} \theta_{2}^{*}} d \theta_{1} \\
& =L \sqrt{\beta^{2}+\sin ^{2} \theta_{2}^{*}}\left(\theta_{1}^{b}-\theta_{1}^{a}\right) \\
& =L \sqrt{\left(\theta_{2}^{b}-\theta_{2}^{a}\right)^{2}+\sin ^{2} \theta_{2}^{*}\left(\theta_{1}^{b}-\theta_{1}^{a}\right)^{2}}
\end{aligned}
$$

It is clear that the new upper limit in (7) is more tighter than the one in (4), an exemple with the Baxter research robot to compare those upper limits is given in Appendix.

\section{B. Spherical Joints Case}

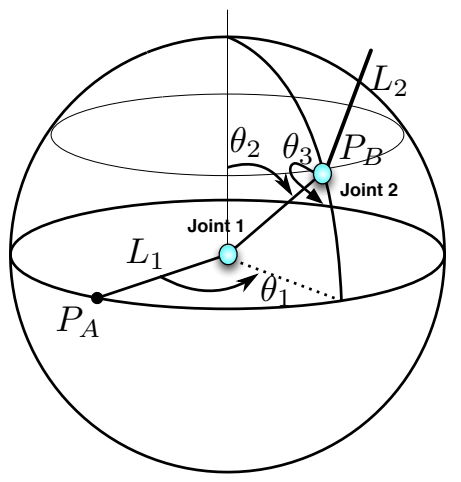

Fig. 4. Displacement of two links controlled by two spherical joints

Another interesting type of joints is the spherical joints, this kind of joints is widely used in computer graphics as well as in robotics where three revolute joints control a given link.

Let us consider the case of two links of length $L_{1}$ and $L_{2}$ respectively, and two spherical joints as shown in Fig. 4. Each spherical joint is represented by three rotations, i.e. joint $1 \triangleq\left[\theta_{1}, \theta_{2}, \theta_{3}\right]^{T}$ and joint $2 \triangleq\left[\theta_{4}, \theta_{5}, \theta_{6}\right]^{T}$, the rotations of joint 2 are not represented in the figure for clarity reasons.

For this linkage, the upper bounds $\bar{\lambda}_{i}(i=1,2)$ can be written as follows:

$$
\begin{aligned}
\bar{\lambda}_{1}= & L_{1} \sqrt{\left(\theta_{2}^{b}-\theta_{2}^{a}\right)^{2}+\sin ^{2} \theta_{2}^{*}\left(\theta_{1}^{b}-\theta_{1}^{a}\right)^{2}} \\
\bar{\lambda}_{2}= & \left(L_{1}+L_{2}\right) \sqrt{\left(\theta_{2}^{b}-\theta_{2}^{a}\right)^{2}+\sin ^{2} \theta_{2}^{*}\left(\theta_{1}^{b}-\theta_{1}^{a}\right)^{2}}+ \\
& L_{2}\left(\theta_{3}^{b}-\theta_{3}^{a}\right)+L_{2} \sqrt{\left(\theta_{5}^{b}-\theta_{5}^{a}\right)^{2}+\sin ^{2} \theta_{5}^{*}\left(\theta_{4}^{b}-\theta_{4}^{a}\right)^{2}}
\end{aligned}
$$

where $\sin \theta_{\times}^{*}, \theta_{\times}^{a}$ and $\theta_{\times}^{b}$ are defined in the same way as in Section III-A.

Note that $\lambda_{1}$ is the same displacement defined in (7), this is because $\theta_{3}$ does not affect the workspace displacement of the first link. On the other hand, $\theta_{3}$ affects the displacement of the second link. This result can be easily extended to the case of an articulated model with $N$ spherical joints.

\section{Minimum Obstacle Distance Computation}

In [10], it has been proven that a minimum clearance $\zeta$ between objects $A_{1}$ and $A_{2}$ at any configuration $q$ between $q_{a}$ and $q_{b}$ is guaranteed if:

$$
\lambda_{1}\left(q_{a}, q_{b}\right)+\lambda_{2}\left(q_{a}, q_{b}\right)<\eta_{12}\left(q_{a}\right)+\eta_{12}\left(q_{b}\right)-2 \zeta
$$

This feature is interesting, however it suffers from several disadvantages, for instance:

1) As the minimum clearance is fixed, the planner might ignore a narrow passage, which would be the only feasible solution.

2) The criterion in (9) does not allow to compare two clear paths. However, in motion planning we are usually interested in sorting admissible paths according to their clearance, this is particularly interesting for safety issue, either for the robot or for a human interacting with the robot.

For a path where two objects $A_{1}$ and $A_{2}$ move between two configurations $q_{a}$ and $q_{b}$, we define the estimated clearance as:

$$
\delta=\frac{\eta_{12}\left(q_{a}\right)+\eta_{12}\left(q_{b}\right)-\lambda_{1}\left(q_{a}, q_{b}\right)-\lambda_{2}\left(q_{a}, q_{b}\right)}{2}
$$

If $A_{2}$ is a fixed obstacle, $\lambda_{2}\left(q_{a}, q_{b}\right)=0$ and (10) becomes:

$$
\begin{aligned}
\delta & =\frac{\eta_{12}\left(q_{a}\right)+\eta_{12}\left(q_{b}\right)-\lambda_{1}\left(q_{a}, q_{b}\right)}{2} \\
& =\operatorname{dist}\left(q_{a}, q_{b}, A_{1}, A_{2}\right)
\end{aligned}
$$

It is worth pointing out that the value of $\delta$ can be either negative or positive:

- $\delta \leq 0$ implies that the motion cannot be guaranteed to be collision-free, this is because (1) is not satisfied. A sub-segmentation is necessary.

- $\delta>0$, then $\delta$ is the minimum distance between $A_{1}$ and $A_{2}$ along the path.

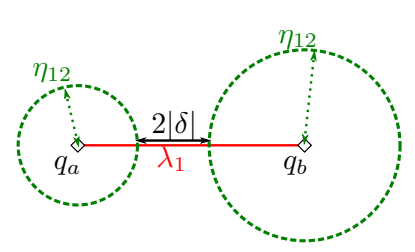

(a) The potential maximum traveled distance by the link is higher than the sum of minimum distance from the object at each configuration $(\delta \leq$ 0)

Fig. 5. Illustration of the relations between distances in the workspace.

A schematic example that illustrates the above two cases is given in Fig. 5. For more details about this representation, refer to [10].

The procedure to compute the minimum clearance along a path segment and sorting collision-free segment paths according to their clearance is given in Algorithm 1. Note that each element of the structure segment refers to a specific 


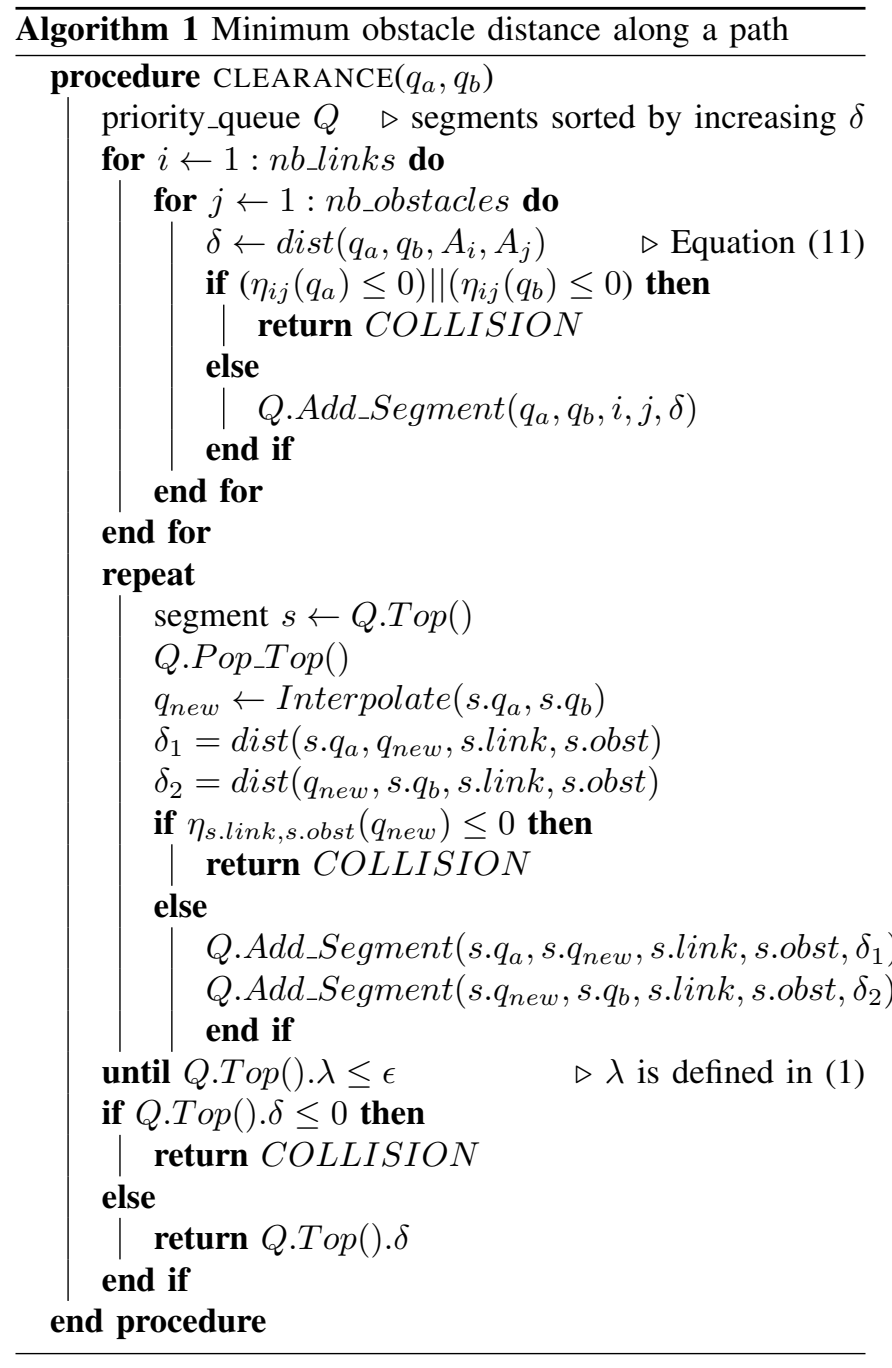

pair of link/obstacle evaluated between two states and is used to store the corresponding distance information. Parameter $\epsilon$ can be defined as the maximum admissible error in the distance estimation. It is a positive user-defined constant that affects the performances of the algorithm: decreasing it improves the returned distance estimation accuracy whereas increasing it reduces the required computational burden to generate the estimation, the impact of $\epsilon$ on the accuracy and the computational complexity is shown in Section V. Table I.

As it has been mentioned in Section II, if the inequality (1) is not satisfied, a sub-segmentation by adding an intermediate state is necessary. However, the location of that state can have a significant impact on the algorithm convergence rate. Many covering strategies have been proposed in [1], however we selected the strategy that samples toward the part of the path that is most likely to be in collision. This strategy yields to a minimum number of segments evaluation, and can be described as follows :

- When $\delta<0$, the new sampled state corresponds to the middle of the uncovered length (Fig. 6(a))

- When $\delta \geq 0$, the new sampled state is the projection of the intersection of the two circles onto the path segment (Fig. 6(b))

We refer to this strategy as the Interpolate $\left(q_{a}, q_{b}\right)$ procedure in Algorithm 1.

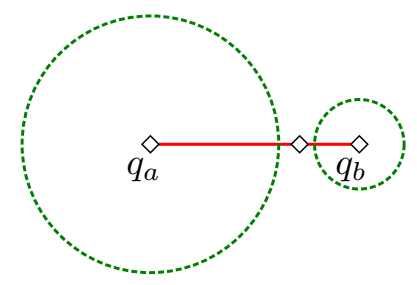

(a) Strategy when $\delta<0$

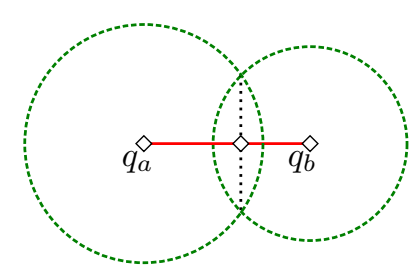

(b) Strategy when $\delta \geq 0$
Fig. 6. Illustration of the covering strategy

Lemma. The estimated and exact distances to obstacles satisfy the following inequality:

$$
\delta_{e x a}-\frac{\epsilon}{2} \leq \delta \leq \delta_{e x a}
$$

where $\delta_{\text {exa }}$ and $\delta$ are, respectively, the exact and estimated minimum distances between two objects $A_{1}$ and $A_{2}$, where $A_{1}$ moves from configuration $q_{a}$ to $q_{b}$ and $A_{2}$ is a fixed obstacle.

Proof: Let $q_{\text {worst }}$ be the configuration, between $q_{a}$ and $q_{b}$, where the distance is minimal.

From (11), we can define the error between the exact value and our estimation as:

$$
\begin{aligned}
\Delta & =\delta_{\text {exa }}-\delta \\
& =\eta_{12}\left(q_{\text {worst }}\right)-\frac{\eta_{12}\left(q_{a}\right)+\eta_{12}\left(q_{b}\right)-\lambda_{1}\left(q_{a}, q_{b}\right)}{2}
\end{aligned}
$$

The proof of Equation (12) can now be constructed as follows:

- $\Delta=0$ is the minimum error:

By definition, (11) gives an underestimation of the minimum clearance along a path segment [1]. That means $\delta_{\text {exa }} \geq \delta_{\text {est }}$ is always verified and thus $\Delta \geq 0$. The value $\Delta=0$ can be obtained in at least one case: when $q_{a}=q_{b}$. That means that, in the best case, our method gives no error on the estimation.

- $\Delta=\frac{\epsilon}{2}$ is the maximum error:

To get the maximum value of $\Delta,(13)$ can be rewritten as follows:

$$
\Delta=\eta_{12}\left(q_{w o r s t}\right)-\frac{\eta_{12}\left(q_{a}\right)+\eta_{12}\left(q_{b}\right)}{2}+\frac{\lambda_{1}\left(q_{a}, q_{b}\right)}{2}
$$

We now have two independent parts in the expression of $\Delta$. As $\eta_{12}$ and $\lambda_{1}$ are always positive, the maximum 
value of $\Delta$ is obtained when the two following conditions are fulfilled:

- $\eta_{12}\left(q_{a}\right)+\eta_{12}\left(q_{b}\right)$ is minimal

- $\lambda_{1}\left(q_{a}, q_{b}\right)$ is maximal

As the minimum clearance is obtained at configuration $q_{\text {worst }}$, that means $\eta_{12}\left(q_{*}\right) \geq \eta_{12}\left(q_{\text {worst }}\right)$ where $*$ is $a$ or $b$.

As defined in Algorithm 1, the stopping criterion of the procedure is $\lambda \leq \epsilon$. That means the maximum value that $\lambda_{1}\left(q_{a}, q_{b}\right)$ can take is $\epsilon$.

When replacing these terms in (14), we get $\Delta=\frac{\epsilon}{2}$. This is the maximum error on the estimated clearance that is obtained in the worst case.

Hence, the condition $\delta_{\text {exa }}-\frac{\epsilon}{2} \leq \delta \leq \delta_{e x a}$ is always satisfied.

\section{EXPERIMENTAL RESULTS}

The efficiency and performance of the described method have been tested in simulation and on the real robot Baxter from Rethink Robotics Inc. Baxter is equipped with two 7-DOF manipulator arms. In this study, we only focus on the control of one arm but our method can be easily extended to also consider both arms simultaneously. Our algorithm has been implemented using the platform MoveIt! [11] that integrates the motion planning library OMPL [12] and communicates via ROS (Robotic Operating System) API interface [13].

\section{A. Distance Approximation Function}

Even though the continuous collision checking method tends to limit the number of distance computations, those operations remain widely called during the planning process. Moreover, computing the exact minimum distance between two complex and concave objects is a computationally expensive operation. Moveit! uses the Flexible Collision Library (FCL) [14] as its primary collision checking library. Besides collisions check, FCL can also compute the distance between two non-overlapping objects.

To accelerate the execution of our algorithm, we developed a computationally efficient distance computation function that gives a reasonable approximation of the distance between a link of the robot and an obstacle. This function is based on simplifying the environment representation by transforming each obstacle into its corresponding oriented bounding box (OBB). The Baxter robot is also approximated by its collision geometry model from the Unified Robot Description Format (URDF) file. Thus, the arms are modeled as cylinders and boxes. Each part of the arm is then subdivided into an optimal number of spheres that encompass the initial volume. Finally, the minimum distance between an obstacle and a link becomes the minimum distance between the OBB and the spheres and, hence, can be easily computed.

\section{B. Numerical Experiments}

The first validation phase of our approach was made through simulated scenarios using the Gazebo simulator [15]

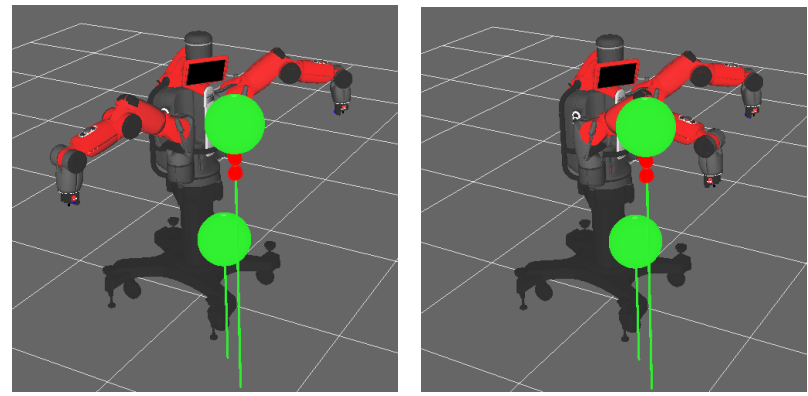

(a) Scenario 1

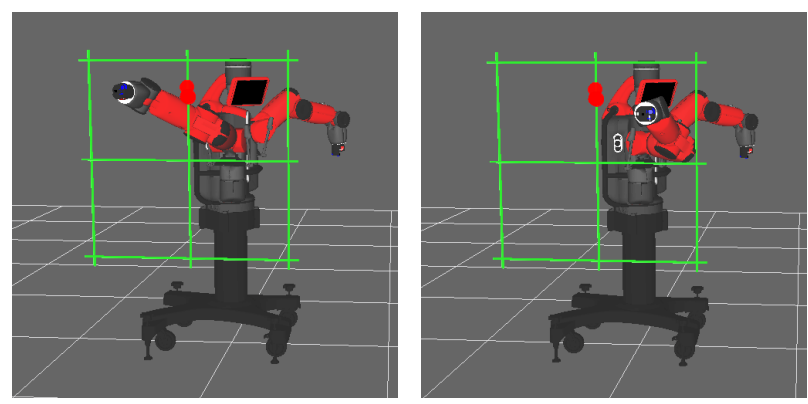

(b) Scenario 2

Fig. 7. Motions that connect near states in the C-space could create a large displacement in the workspace. Discrete collision checker does not detect collisions (represented by red spheres) with the thin rod.

as an interface for the Baxter robot. All experiments are performed on Intel(R) Core(TM) i5-3470 CPU @ $3.20 \mathrm{GHz}$ PC with 8 GB RAM.

Two scenarios have been considered in this study:

- Scenario 1: The right arm is queried to reach successively two particular configurations for the joints. These states are located on the sides of two balloons tethered to the ground by thin rods as shown in Fig. 7(a).

- Scenario 2: The right arm's end-effector is planned to reach specific poses (position and orientation) passing through a wire grid leaving only narrow free spaces see Fig. 7(b).

The conventional method of uniformly discretizing a path to check if it is collision-free is called hereafter the discrete method and our implementation as continuous method.

We implemented the continuous and discrete collision checking methods into the classical RRT* and their performances in finding the minimum distance from obstacles along a path is compared in in Table I. The tests have been carried out in the two scenarios to point out the environmental influence. Each single test gathers 100 motion planning tasks, and the allowed time for task planning is set to 20 seconds for both implementations. The standard distance function (FCL) has been used in both cases, and for different values of $\epsilon$. For the discrete case, $\epsilon$ is the sampling rate in $\mathrm{C}$-space. It refers to the length of the longest segment that does not require state validation and is defined as a fraction of the space maximum extent. For the continuous method, $\epsilon$ is the maximum acceptable error in the distance evaluation between two objects.

It is worth pointing out that, for a single arm of Baxter 
robot, the maximum extent in the C-space is approximately $10 \pi$ (the sum of the joint ranges). Thus, for a standard value $\epsilon=5 \%$, a segment will be considered as collision free if the total corresponding joint variation is under $\pi / 2$. In the worst case, this variation can produce a displacement of more than $1,7 \mathrm{~m}$. Conversely, if we want to ensure a maximum error of $10 \mathrm{~mm}$ in the workspace, as done with the continuous method, we have to set a value of $\epsilon=0.03 \%$. This is not an efficient solution as the computational time will tremendously increase.

The distance obtained by the discrete method, with an extremely low value of $\epsilon(0.01 \%)$, is taken as a reference. We indicate by $\Delta$ the error between the reference and the estimated distance.

The sign of $\Delta$ plays a major role with respect to the safety issue. As it is always positive for the continuous method, we ensure that no collision could occur.

The computation time is only indicative because the accuracy of both methods is not comparable. Note that the use of the distance approximation function can tremendously improve the computational time compared to FCL (with an average time gain of 74.6 times in Sc.1 and 5.5 times in Sc.2). However, this strategy underestimates the distance between the objects and might add a non-negligible error on the evaluation $(7.1 \mathrm{~mm}$ for Sc. 1 and $52.5 \mathrm{~mm}$ for Sc. 2 on average). This is a good option when planning in relatively uncluttered areas because it is very fast to compute. On the other hand, that approximation might neglect narrow passages as they could be considered in collision.

TABLE I

RESULTS FOR MINIMUM DISTANCE COMPUTATION ALONG A MOTION

\begin{tabular}{|c|c|c|c|c|c|c|}
\hline Sc. & Method & $\epsilon$ & $\begin{array}{c}\operatorname{mean}(\Delta) \\
(\mathrm{mm})\end{array}$ & $\begin{array}{c}\min (\Delta) \\
(\mathrm{mm})\end{array}$ & $\begin{array}{c}\max (\Delta) \\
(\mathrm{mm})\end{array}$ & $\begin{array}{c}\text { Avg. time } \\
(\mathrm{ms})\end{array}$ \\
\hline \hline \multirow{4}{*}{1} & \multirow{2}{*}{ Discrete } & $5 \%$ & -2.0 & -55.3 & 0.0 & 21.06 \\
& & $1 \%$ & -0.2 & -3.2 & 0.0 & 90.30 \\
\cline { 2 - 7 } & \multirow{2}{*}{ Continuous } & $100 \mathrm{~mm}$ & 25.1 & 0.0 & 48.9 & 25.23 \\
& & $10 \mathrm{~mm}$ & 2.6 & 0.0 & 5.0 & 48.69 \\
\hline \hline \multirow{4}{*}{2} & \multirow{2}{*}{ Discrete } & $5 \%$ & -3.1 & -111.8 & 0.0 & 5.15 \\
& & $1 \%$ & -0.2 & -11.4 & 0.0 & 22.57 \\
\cline { 3 - 7 } & \multirow{2}{*}{ Continuous } & $100 \mathrm{~mm}$ & 25.2 & 0.0 & 49.8 & 3.96 \\
& & $10 \mathrm{~mm}$ & 2.5 & 0.0 & 4.9 & 5.60 \\
\hline
\end{tabular}

Table II presents the failure rates as a result of an undetected collision. We chose a fixed value $\epsilon=10 \mathrm{~mm}$ for the continuous collision checker to enable the robot to operate in a complex environment, like the one in Scenario 2.

As anticipated, the continuous method did not omit any collision and enabled the planner to achieve all tasks. On the other hand, the discrete method tends to find inadmissible solutions due to undetected collisions.

We have also validated the experiment in Scenario 1 on the real Baxter robot as shown in Fig. 8. The whole experiment can be seen in the accompanying video.
TABLE II

TASK VALIDITY COMPARISON

\begin{tabular}{|c|c|c|c|}
\hline Sc. & Method & $\epsilon$ & Failure \\
\hline \hline \multirow{2}{*}{1} & \multirow{2}{*}{ Discrete } & $5 \%$ & $100 \%$ \\
\cline { 3 - 4 } & & $1 \%$ & $17 \%$ \\
\cline { 3 - 4 } & Continuous & $10 \mathrm{~mm}$ & $0 \%$ \\
\hline \hline \multirow{2}{*}{2} & \multirow{2}{*}{ Discrete } & $5 \%$ & $96 \%$ \\
\cline { 3 - 4 } & & $1 \%$ & $48 \%$ \\
\cline { 2 - 4 } & Continuous & $10 \mathrm{~mm}$ & $0 \%$ \\
\hline
\end{tabular}

\section{CONCLUSION}

This paper presented a new formulation to bound the motions of spherical and two revolute joints. This formulation is integrated into the bisection collision checking method [1] in order to provide tighter motion bounds than the original formulation. An extension to get the minimum distance to obstacles is also presented, this extension can be directly integrated into any sampling-based motion planning algorithm to sort collision-free paths according to their clearance.

Our experiments show that the computational time for the new implementation of bisection collision checking method is comparable or faster than the conventional discrete method, most importantly it never miss a potential collision with the environment. For those reasons, it can be an appropriate candidate to remplace the conventional discrete method in environments with thin objects.

Future work will focus on integrating our implementation of the bisection collision checking method into a motion planning algorithm to:

- Exploit the feature of minimum distance to obstacles to maximize human safety in the context of human-robot cooperation.

- Deal with bi-manipulation tasks by integrating the second arm of the Baxter research robot.

\section{APPENDIX}

To compare the upper limits in (7) and (4), we consider the shoulder's two revolute joints (joints 1 and 2 in Fig. 9) of the Baxter robot arm, and the following initial and final configurations:

$$
\begin{aligned}
& \theta_{1}^{a}=-\frac{\pi}{2}, \theta_{1}^{b}=\frac{\pi}{3} \\
& \theta_{2}^{a}=-\frac{\pi}{3}, \theta_{2}^{b}=\frac{\pi}{3}
\end{aligned}
$$

The arm length starting from the axe of joint 1 is $L=$ $0.9679 \mathrm{~m}$.

In this cas, the upper limits are:

$$
\begin{aligned}
\bar{\lambda} & =3.4997 m \\
\bar{\lambda}^{*} & =5.3213 m \\
\Delta \lambda & \triangleq \bar{\lambda}^{*}-\bar{\lambda}=1.8216 m
\end{aligned}
$$




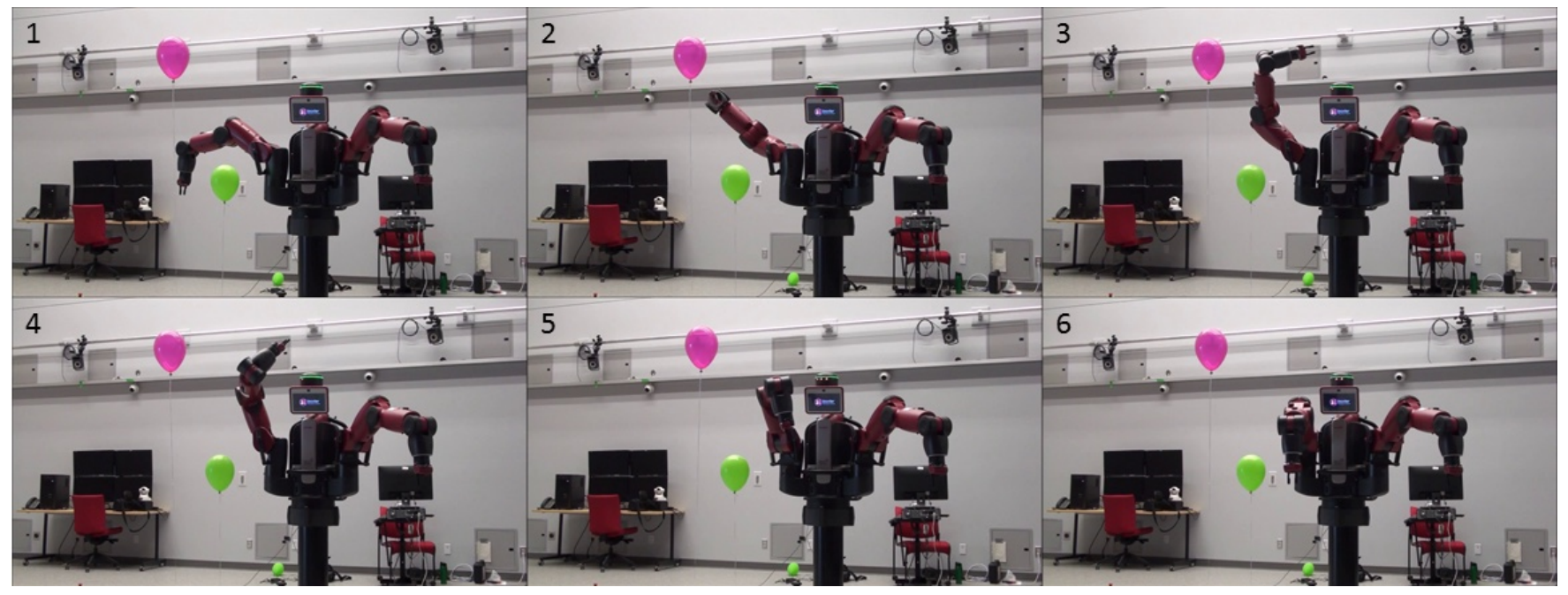

Fig. 8. Snapshots of the Baxter robot executing a motion planning in an unstable environment using our implementation of the continuous collision checking method.

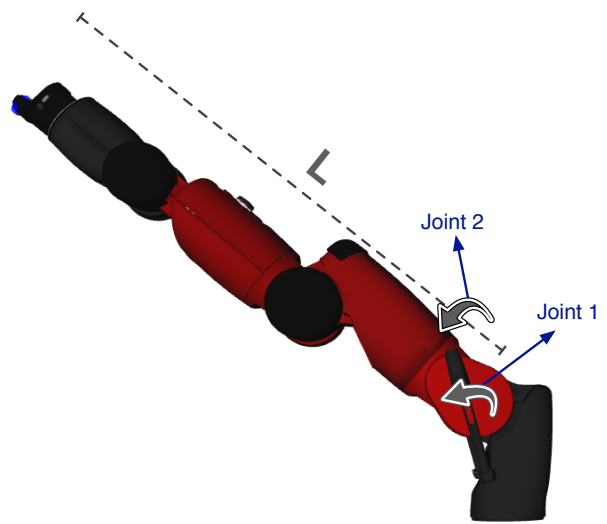

Fig. 9. Baxter research robot arm.

where $\bar{\lambda}$ and $\bar{\lambda}^{*}$ are the upper limit computed by (7) and (4) respectively.

The above results point out the significant difference between the two upper limits on the workspace displacement, and that our new formulation provides a tighter bound on the displacement than the original one.

\section{ACKNOWLEDGMENT}

This research is supported by the Natural Sciences and Engineering Research Council of Canada (NSERC) under the grant RGPIN-2012-419406.

\section{REFERENCES}

[1] F. Schwarzer, M. Saha, and J.-C. Latombe, "Adaptive dynamic collision checking for single and multiple articulated robots in complex environments," IEEE Transactions on Robotics, vol. 21, no. 3, pp. 338-353, 2005.

[2] S. Quinlan, "Real-time modification of collision-free paths," Ph.D. dissertation, Stanford University, 1994.
[3] N. Vahrenkamp, T. Asfour, and R. Dillmann, "Efficient motion planning for humanoid robots using lazy collision checking and enlarged robot models," in IEEE/RSJ International Conference on Intelligent Robots and Systems, 2007, pp. 3062-3067.

[4] N. Vahrenkamp, P. Kaiser, T. Asfour, and R. Dillmann, "Rdt+: A parameter-free algorithm for exact motion planning," in IEEE International Conference on Robotics and Automation (ICRA), 2011, pp. 715-722.

[5] S. Redon, M. C. Lin, D. Manocha, and Y. J. Kim, "Fast continuous collision detection for articulated models," Journal of Computing and Information Science in Engineering, vol. 5, no. 2, pp. 126-137, 2005.

[6] X. Zhang, S. Redon, M. Lee, and Y. J. Kim, "Continuous collision detection for articulated models using taylor models and temporal culling," ACM Trans. Graph., vol. 26, no. 3, July 2007.

[7] M. Tang, R. Tong, Z. Wang, and D. Manocha, "Fast and exact continuous collision detection with bernstein sign classification," ACM transactions on graphics, vol. 33, no. 6, p. 186, 112014.

[8] M. Tang, Y. J. Kim, and D. Manocha, "C2A: Controlled conservative advancement for continuous collision detection of polygonal models,' in IEEE International Conference on Robotics and Automation, May 2009, pp. 849-854.

[9] S. Redon and M. C. Lin, "Practical local planning in the contact space," in IEEE International Conference on Robotics and Automation, April 2005, pp. 4200-4205.

[10] F. Schwarzer, M. Saha, and J.-C. Latombe, "Exact collision checking of robot paths," in Algorithmic foundations of robotics $V$. Springer, 2004, pp. 25-41.

[11] I. A. Sucan and S. Chitta, "Moveit!" 2018. [Online]. Available: http://moveit.ros.org

[12] I. A. Şucan, M. Moll, and L. E. Kavraki, "The Open Motion Planning Library," IEEE Robotics \& Automation Magazine, vol. 19, no. 4, pp. 72-82, December 2012, http://ompl.kavrakilab.org.

[13] M. Quigley, K. Conley, B. Gerkey, J. Faust, T. Foote, J. Leibs, R. Wheeler, and A. Y. Ng, "ROS: an open-source robot operating system," in ICRA workshop on open source software, vol. 3, no. 3.2, 2009 , p. 5.

[14] J. Pan, S. Chitta, and D. Manocha, "FCL: A general purpose library for collision and proximity queries," in IEEE International Conference on Robotics and Automation (ICRA), 2012, pp. 3859-3866.

[15] N. Koenig and A. Howard, "Design and use paradigms for gazebo, an open-source multi-robot simulator," IEEE/RSJ International Confer2004. 